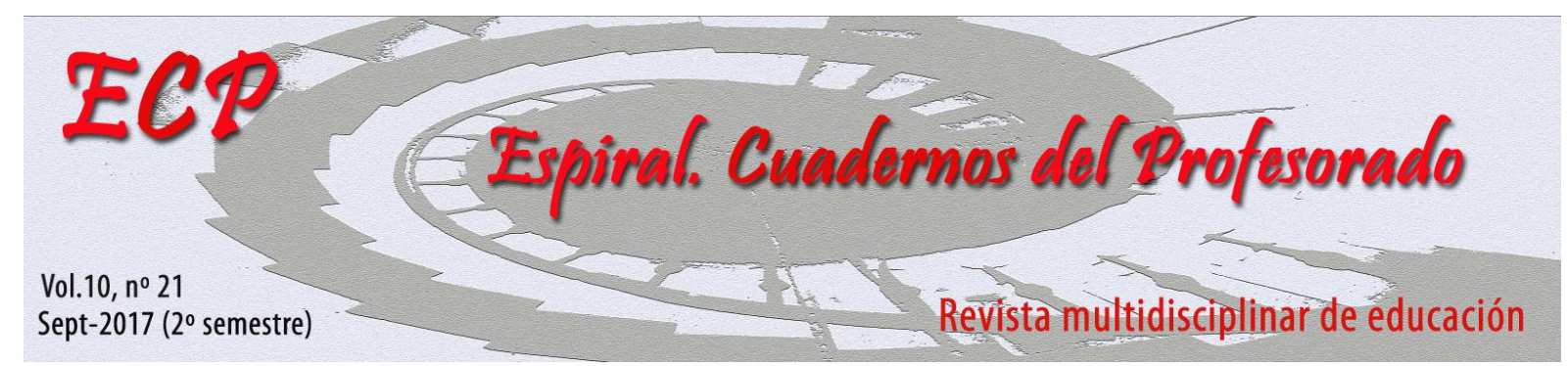

\title{
Satisfacción laboral de trabajadores de actividades deportivas y recreativas en turismo activo
}

Job satisfaction of workers of sports and recreational activities in active tourism

\section{Moisés Grimaldi-Puyana ${ }^{1,3}$, Antonio Jesús Sánchez-Oliver ${ }^{1,2}$, Virginia Alcaraz-Rodríguez ${ }^{1,2}$, M Marta Pérez Villalba ${ }^{3}$}

1. Universidad de Sevilla

2. Universidad Pablo Olavide

3. Grupo de investigación Gestión e Innovación en Servicios Deportivos, Ocio, Recreación y Acción Social (GISORAS), Universidad de Sevilla

RESUMEN: La satisfacción laboral es un estado emocional sobre la percepción subjetiva de las experiencias laborales de los trabajadores, que afecta directamente en la calidad del servicio prestado. Por ello, El objetivo del estudio analizar la satisfacción laboral de los trabajadores de turismo activo y sus condiciones de trabajo, y el efecto de estas en su satisfacción laboral en la comunidad autónoma de Andalucía. Para determinar los niveles de satisfacción laboral, se utilizó la herramienta de "Overall Job Satisfaction", de fácil uso y recomendada por el Ministerio de Trabajo y Asuntos Sociales del Estado español. La muestra estuvo compuesta por 218 profesionales del sector del turismo activo andaluz, con una media de edad de 34 años, (73,8\% hombres y 26,2\% mujeres). De los resultados del presente estudio, podemos concluir que los trabajadores de turismo activo en Andalucía, a modo general, están satisfechos, ejercen su profesión por cuenta propia y son mayoritariamente hombres. Del mismo modo, también podemos decir que la satisfacción de estos trabajadores no guarda relación con las siguientes condiciones laborales: actividad desempeñada en la organización, tipo de contrato, realización de otras funciones propias de la actividad, horas de dedicación semanal o el sueldo percibido.

Palabras clave: Ocio; deporte; turismo; trabajador; satisfacción.

ABSTRACT: Job satisfaction is an emotional state on the subjective perception of workers' work experiences, which directly affects the quality of the service provided. Therefore, the objective of this study is to analyze the labor satisfaction of active tourism workers and their working conditions, and the effect of these on their job satisfaction in the Autonomous Community of Andalusia. To determine the levels of job satisfaction, the "Overall Job Satisfaction" tool, which was easy to use and recommended by the Spanish Ministry of Labor and Social Affairs, was used. The sample consisted of 218 professionals from the Andalusian active tourism sector, with a mean age of 34 years (73.8\% men and $26.2 \%$ women). From the results of the present study, we can conclude that the active tourism workers in Andalusia, in general, are satisfied, practice their profession on their own and are mostly men. In the same way, we can also say that the satisfaction of these workers is not related to the following working conditions: activity performed in the organization, type of contract, performance of other functions of the activity, hours of weekly dedication or the salary received.

Key words: Leisure; sport; tourism; employee; job satisfaction.

Grimaldi-Puyana, M., Sánchez-Oliver, A. J., Alcaraz-Rodríguez, V., y Pérez Villalba, M. (2017). Satisfacción laboral de trabajadores de actividades deportivas y recreativas en turismo activo. Espiral. Cuadernos del Profesorado, 10(21), 140-148.

Fecha de recepción: 12/05/2017

Fecha de aceptación: 17/07/2017
Correspondencia: mgrimaldi@us.es

(M. Grimaldi-Puyana) 


\section{Introducción}

Actualmente podemos ver como la práctica deportiva en España sigue aumentado, según los datos ofrecidos por el Consejo Superior de Deportes (2015) el incremento supone un 9,2\% sobre la encuesta realizada en 2010. Este crecimiento se ha producidos desde 1980 hasta la década pasada, donde se ha producido un aumento del consumo de productos y servicios de actividad física deportiva (Barranco, Grimaldi-Puyana, Crovetto, Barbado, Boned, y Felipe, 2015). En el mismo sentido las actividades turísticas deportivas en el medio natural y espacios naturales protegidos son una realidad de tendencia consolidada (García-Sauna, 2010), los datos de la encuesta de hábitos deportivos en Española sitúan el "senderismo y montañismo" en tercera posición, por detrás del ciclismo y la natación.

Dicha tendencia se viene observando desde principios de siglo, encaminada hacia lo natural, ecológico y deportivo, de ahí por el creciente interés de la práctica deportiva en el medio natural (Luque-Gil, 2003; Salguero-Pérez, 2002). Estas tendencias del mercado, ayuda a crear nuevas oportunidades para emprender, dirigir o ampliar negocios (Sánchez Oliver, García Fernández, Grimaldi-Puyana, y Feria-Madueño, 2017).

En este sentido el binomio turismo y deporte en el medio natural o Turismo Activo (TA), se presenta como una extraordinaria combinación afectiva empresarial de carácter comercial, con amplio mercado de futuro y éxito esperanzador (González-Molina, 2008), que trata de dar respuesta a la demandada que exige nuevas actividades, que sean capaces de generar ambientes con un clima de amistad, compañerismo, cooperación y superación personal.

Dicha tendencia ha producido nuevos yacimientos de empleo dentro del ocio-recreación deportivo (Pérez, M., Vilanova, y Grimaldi-Puyana, 2016), aunque sin poder afirmar que estos se hayan acompañado de una adecuada legislación laboral (García, Lago, y Fernández, 2011; GrimaldiPuyana, 2015). Este hecho ha supuesto que las condiciones laborales de estos profesionales sean en muchos casos precarias, sin valorar la formación o experiencia de los trabajadores (Gambau, 2011), dado que una buena práctica en gestión de recursos humanos, debe ir orientada a la actualización en función del contexto social y laboral en el que se encuentren, que adapte la totalidad de los perfiles profesionales.

Desde el punto de vista de la seguridad, las actividades deportivas en el medio natural, llevan implícita un elevado riesgo, incluso más que en cualquier otra práctica deportiva (Grimaldi-Puyana, Felipe, y Cuadrado-Reyes, 2013). De la misma forma Gutiérrez-Pérez (2007) en su capítulo "competencias del monitor de naturaleza" señala la importancia de la figura del monitor de la naturaleza, con la importancia en "la cualificación profesional y dominio conceptual actitudinal y de capacidades". Por ello, para garantizar un servicio de ocio, tiempo libre de TA de calidad, la clave es contar con el personal cualificado y satisfecho laboralmente (Grimaldi-Puyana, 2015; GrimaldiPuyana, Fernández-Gavira, y García-Fernández, 2013).

Sin embargo no es fácil definir el concepto de satisfacción laboral, podemos decir que se trata de un estado emocional positivo sobre la percepción subjetiva de las experiencias laborales de los trabajadores (Alonso, 2008; Aamont, 2010; Robbins, 2004; Sánchez, 2006; Casas, Repullo, Lorenzo y Cañas, 2002). Además es de interés desde los años sesenta del siglo pasado, y del que se ha producido un aumento en investigaciones en diferentes áreas (Anaya y Suárez, 2004; Martín, Campos-Izquierdo, Jiménez y Martínez, 2007), sin embargo son escasas en el sector deportivo (Sánchez-Alcaraz y ParraMeroño, 2013; Sánchez-Alcaraz, Gómez, Parra y García, 2014).

Es obvio que el conocimiento de la organización como modelos de gestión, tipo de organizaciones, tendencias, trabajadores, y satisfacción laboral, puede ayudar a conseguir una ventaja competitiva. No obstante, su generación no garantiza eficiencia y efectividad para la organización, por lo que la gestión del conocimiento adquirido tendrá la misión de adaptarlo para el logro de los objetivos marcados por la misma (Theriou y Chatzoglou, 2008). Como señala Sánchez-Alcaraz (2012) en su estudio sobre la satisfacción laboral de los socorristas de playa, "Disponer de personas motivadas y satisfechas con su trabajo aumenta claramente su rendimiento y la calidad del servicio que prestan y disminuyen el absentismo, la accidentalidad y la posibilidad de cambio o abandono de 
la organización”. Por tanto, en el sector deportivo del TA en particular, también el conocimiento de la satisfacción laboral se sitúa como una variable decisiva en la gestión de recursos humanos, tal y como indican Bernabé, Campos-Izquierdo y González (2016) con una relación directa con la calidad del servicio.

Dado que el técnico deportivo es la cara visible y representa la estrategia empresarial, al estar en contacto directo con el cliente, si estos no se encuentran motivados, satisfechos y fidelizados, les resultará más difícil mantener al cliente, así como atraer a potenciales consumidores (Sánchez-Alcaraz y Parra-Meroño, 2013b). Por este motivo, se planteó como objetivo del estudio analizar la satisfacción laboral de los trabajadores TA y sus condiciones de trabajo, y el efecto de estas en su satisfacción laboral en la comunidad autónoma de Andalucía.

\section{Método}

\section{Muestra}

Como podemos observar en la Tabla 1, la muestra estuvo compuesta por 218 profesionales del sector del TA de Andalucía (edad media: $34,14 \pm 6,80$ años). De los que el 73,8\% eran hombres y el $26,2 \%$ mujeres, de estos el 55,3\% manifestó estar casado o era pareja de hecho, mientras que el 34,2\% estaban solteros y vivían en parejas $10,5 \%$. Respecto a las funciones que desempeñaban, el $54,1 \%$ manifestó hacer funciones de director técnico y monitores a la vez (DTM), el 26,6\% desempeñaban funciones de director técnico solamente (DT) y el 19,3\% funciones de monitor o instructor solamente (MI).

Tabla 1.

Distribución de la muestra en función del género, nivel de estudios y cargo desempeñado $(n=218)$

\begin{tabular}{llc}
\hline & & $\%$ \\
\hline \multirow{2}{*}{ Género } & Hombres & 73,8 \\
& Mujeres & 26,2 \\
\hline \multirow{3}{*}{ Cargo que desempeña } & DTM & 54,1 \\
& DT & 26,6 \\
& MI & 19,3 \\
\hline \multirow{3}{*}{ Nivel de estu- } & Sin titulación TA & 34,2 \\
dios & TAFAD & 13,2 \\
& Magisterio & 21,1 \\
& Graduado CCAFD & 7,9 \\
& Grado Medio & 10,5 \\
& Técnico deportivo & 13,2 \\
\hline
\end{tabular}

Nota. Director técnico y monitores a la vez = DTM; director técnico; monitor instructor.

\section{Instrumentos}

Para determinar los niveles de satisfacción laboral, utilizamos la herramienta de Overall Job Satisfaction (Warr, Cook, y Wall, 1979), se trata de una herramienta de fácil uso y recomendada por el Ministerio de Trabajo y Asuntos Sociales del Estado español. La herramienta nos muestra tres apartados, satisfacción intrínseca (reconocimiento obtenido en el trabajo, responsabilidad, aspectos relativos al contenido de la tarea, etc.), satisfacción extrínseca (organización del trabajo, horario, remuneración, condiciones físicas del trabajo, etc.) y satisfacción total. Esta herramienta presenta una escala Likert, con siete grados de satisfacción, siendo "1 muy insatisfecho; 2 insatisfecho; 3 moderadamente insatisfecho; 4 indiferente; 5 moderadamente satisfecho; 6 satisfecho; 7 muy satisfecho. Por último, los valores de fiabilidad de las escalas según el Alfa de Crobach que se obtuvieren en el presente estudio (0,92 en satisfacción laboral intrínseca, 0,81 en satisfacción laboral 
extrínseca), se situaron por encima de los valores proporcionados en el estudio original en sus dos pruebas originales (Tabla 2) y en valores parecidos a los de satisfacción laboral total $(0,83)$.

Tabla 2.

Valores del Alfa de Cronbach del estudio original y el presente estudio

\begin{tabular}{lccc} 
Dimensiones & $\begin{array}{c}\text { Estudio 1 original de Warr Estudio 2 original de Warr } \\
(n=200)\end{array}$ & Estudio actual \\
& 0,79 & 0,88 & \\
\hline Satisfacción Intrínseca & 0,74 & 0,78 & 0,92 \\
Satisfacción Extrínseca & 0,85 & 0,88 & 0,81 \\
Satisfacción total & & 0,83 \\
\hline
\end{tabular}

Nota. Tabla de elaboración propia basado en Warr, Cook, \& Wall (1979).

La recogida de datos se realizó a través de un cuestionario on-line, que se llevó a cabo gracias a la colaboración de distintas organizaciones relacionadas con el sector de TA en Andalucía. Los participantes recibían un mensaje en su bandeja de correo electrónico informando del propósito del estudio, de las características del instrumento y del acceso al cuestionario mediante un enlace a una dirección URL. Los participantes contestaban a las preguntas en la misma aplicación y al terminar, esta información era volcada de forma automática en una base de datos en el programa estadístico SPSS versión 21.

\section{Análisis de datos}

Todas las variables fueron sometidas al contraste de normalidad Kolomogorov-Smirnov, los resultados mostraron que la muestra no seguía una distribución normal. Se realizaron pruebas de análisis descriptivo (medias, desviaciones típicas y frecuencias) e inferencial (correlación de Pearson entre variables de carácter numérico y siguiendo las indicaciones de Sierra, 2001). Además se realizaron pruebas no paramétricas para las variables independientes (U de Mann-Withney), y si la variable independiente presentaba más de dos categorías se utilizó la prueba de $\mathrm{H}$ de Kruskal-Wallis. Para el análisis de los valores esperados y observado en variables nominales se realizó la prueba de Chi-cuadrado. Y por último, se estableció un nivel de confianza del 95\% y un nivel de significación estadística de $p<0,05$.

\section{Resultados}

En primer lugar, respecto a la dedicación semanal observamos un porcentaje superior en hombres, con contratos de jornada completa frente a los contratos de jornada parcial de las mujeres estadísticamente significativos mujeres $(p<0,05)$. El 44,8\% de los hombres se encuentran en situación de jornada completa frente al $22,2 \%$ de mujeres, mientras que en situación de contratación parcial estos datos son favorables a la mujer con un $77,8 \%$ frente al $55,2 \%$ de hombres contratados parcialmente. Sin embargo no se aprecian diferencias salariales estadísticamente significativas entre hombres y mujeres. Por lo que podemos ver que el 13,8\% de trabajadores se encuentran en un rango salarial <500€; 44,7\% entre 501-1.000€; $21,1 \%$ entre $1.001-1.500 €$ y un $18,4 \%$ entre $1.5001-2000 €$.

En segundo lugar observamos un elevado número de trabajadores que trabajan por cuenta propia en el sector del TA, dado que $68,4 \%$ ejercen su profesión como autónomos, mientras que el $31,6 \%$ lo realiza por cuenta ajena (13,2\% indefinidos por temporadas, $10,5 \%$ indefinidos y $7,9 \%$ contratos por obras y servicios). Tampoco se aprecian diferencias estadísticamente significativas en cuanto al género se refiere. Sin embargo, existen diferencias estadísticamente significativa en cuanto al a la relación género y el tipo de horario $(p<0,01)$. Los hombres con un $41,4 \%$ frente al $11,1 \%$ de mujeres indican tener horario flexible, mientras que ambos casos manifiestan tener horarios a demanda del cliente (hombres 44,4\%; mujeres 37,9\%).

Al analizar la titulación, el 34,2\% de los encuestados manifestaron no tener titulación relacionado el anexo VI del Decreto 22/2002, de 26 de enero, de turismo en el medio rural y turismo activo, frente al $65 \%$ que indican alguna titulación recogida en la normativa, como un $21,1 \%$ de 
titulados en Magisterios de Educación Física, 13,2\% Técnico Superior en Animación Físico deportiva, 13,2\% Técnico Deportivo relacionado con a la modalidad deportiva, 10,5\% Grado Medio de conducción en Actividades en el Medio Natural y 7,9\% de Titulados en Ciencias de la Actividad Física y el Deporte.

Como podemos ver en la Tabla 3, se describen los resultados de satisfacción laboral de los profesionales del sector del TA de Andalucía, en la dimensión intrínseca, extrínseca y satisfacción laboral total. Los profesionales del sector deportivo en el medio natural, muestran valores promedio más altos de satisfacción extrínseca $(M=43,1 ; \mathrm{DT}=7,8)$ que satisfacción intrínseca $(M=43,1$; DT=10,6). Estos valores adaptados a la escala (1-7) en herramienta utilizada, situaría a los trabajadores en la dimensión intrínseca en "moderadamente satisfecho" $(5,92)$, mientras que sitúa en "satisfecho" a las otras dos dimensiones extrínseca $(6,15)$ y satisfacción total $(6,03)$.

Tabla 3.

Valores de Satisfacción Laboral de los trabajadores de TA.

\begin{tabular}{lcccc}
\hline Dimensiones & Media & DT & Mín & Máx \\
\hline Satisfacción Intrínseca & 41,47 & 7,81 & 38,91 & 44,12 \\
Satisfacción Extrínseca & 43,10 & 10,65 & 39,62 & 46,61 \\
Satisfacción total & 84,57 & 17,29 & 78,89 & 90,26 \\
\hline
\end{tabular}

Nota. Valores expresados en Medias; desviación típica (DT); Límite mínimo (Mín); Límite Máximo (Máx); correlación estadísticamente significativa bilateral al nivel $0,01(* *)$.

Se realizaron los análisis de las sub-escalas de satisfacción laboral con las variables sociodemográficas. En primer lugar al comparar el género con la satisfacción laboral, no existían diferencias estadísticamente significativas en la satisfacción laboral intrínseca $(U=81.500 ; p<0,093)$, satisfacción laboral extrínseca $(U=119.500 ; p<0,711)$ ni satisfacción laboral total $(U=99.500 ; p<0,293)$ por razones de sexo. De la misma forma al analizar las puntuaciones en función de su estado civil, no se encontraron diferencias estadísticamente significativas al comparar la satisfacción laboral intrínseca $(K=3,16 ; p<0,367)$, satisfacción laboral extrínseca $(K=4,03 ; p<0,258)$ ni satisfacción laboral total $(K=4,07 ; p<0,253)$. Tampoco se aprecian diferencias estadísticamente significativas al comparar el nivel de formación alcanzado con la satisfacción laboral intrínseca $(K=9,967 ; p<0,076)$, satisfacción laboral extrínseca $(K=6,762 ; p<0,239)$ ni satisfacción laboral total $(K=7,553 ; p<0,183)$.

A continuación podemos ver las variables vinculadas a las características de los puestos de trabajo y condiciones laborales. No se observaron diferencias estadísticamente significativas de satisfacción laboral por razones de la actividad que desempeña en la organización (DTM, DT y MI), tipo de contrato (autónomo, indefinido, obra y servicio y fijo discontinuo), por razón de las funciones que desempeñan además de las que fueron contratados o las propia de las actividad deportiva (redes sociales, marketing, gestión administrativa, etc.), horas a la semana (hasta 10 horas semana, de 10 a 20 horas semana, 21 a 30 horas y 31 a 40 horas semana), o sueldo percibido al mes (fijo o variable), que mejoren la satisfacción laboral de los trabajadores.

En la tabla 4, podemos ver los valores medios que relacionan la satisfacción laboral con el ámbito, experiencia laboral y tipo de jornada. Observamos valores medios superiores en trabajadores de jornada completa, frente a los de jornada parcial. Los trabajadores de jornada completa se muestran "satisfecho" en la satisfacción intrínseca $(K=9,561 ; p<0,008)$ y en satisfacción laboral total $(K=6,5522 ; p<0,038)$, por el contrario en la satisfacción laboral extrínseca no se aprecian diferencias estadísticamente significativas $(K=4,912 ; p<0,086)$. Respecto a los trabajadores a jornada parcial, su grado de satisfacción laboral intrínseco y total fue "moderadamente satisfecho", en la satisfacción intrínseca $(K=9,561 ; p<0,008)$ y en satisfacción laboral total $(K=6,5522 ; p<0,038)$, mientras que en la satisfacción laboral extrínseca no se aprecian diferencias estadísticamente significativas $(K=4,912$; $p<0,086$ ).

En la relación de satisfacción laboral y ámbito (público y privado), se observan diferencias estadísticamente significativas en satisfacción intrínseca $(U=52,500 ; p<0,05)$ y en la satisfacción laboral total $(U=50,000 ; p<0,05)$. Situando los valores medios superiores en satisfacción intrínseca a 
favor de trabajadores del ámbito privado $(M=6,01$; DT=1,067), frente a los que trabajan en el ámbito público $(M=5,41 ; \mathrm{DT}=1,405)$. Así como valores superiores medios en satisfacción total en trabajadores en ámbito privado $(M=5,42 ; \mathrm{DT}=1,19)$, frente a trabajadores del público $(M=5,5$; DT=1,02).

También se observan diferencias estadísticamente significativas en la relación entre satisfacción laboral y años de experiencia. Situando en valores medios superiores como "satisfechos" en satisfacción intrínseca $(M=6,5 ; p<0,05)$, extrínseca $(M=6,1 ; p<0,05)$ y satisfacción total $(M=6,2$; $p<0,05)$ a los trabajadores con experiencia entre cuatro y siete años. Mientras que los trabajadores con experiencia inferior a tres años y superior a ocho, muestran valores medios de satisfacción laboral de moderadamente satisfechos.

Tabla 4.

Relación entre variable satisfacción laboral con el ámbito, experiencia y Jornada.

\begin{tabular}{cccccccc}
\hline \multirow{2}{*}{ Tipo } & Características & \multicolumn{2}{c}{$\begin{array}{c}\text { Satisfacción } \\
\text { Intrínseca }\end{array}$} & \multicolumn{2}{c}{$\begin{array}{c}\text { Satisfacción } \\
\text { Extrínseca }\end{array}$} & \multicolumn{2}{c}{$\begin{array}{c}\text { Satisfacción } \\
\text { Total }\end{array}$} \\
\cline { 3 - 8 } & & $M$ & $D T$ & $M$ & $D T$ & $M$ & $D T$ \\
\hline \multirow{2}{*}{ Ámbito } & Público & 5,41 & 1,4 & 5,41 & 1,4 & 5,51 & 1,02 \\
& Privado & 6,01 & 1,06 & 5,29 & 1,08 & 6,42 & 1,19 \\
\hline \multirow{3}{*}{ Experiencia } & $<1$ año a 3 años & 6,5 & 1,96 & 6,1 & 4,8 & 6,2 & 0,89 \\
& De 4 años a 7 años & 5,6 & 1,96 & 5,15 & 0,48 & 5,38 & 1,02 \\
& De 8 años a + 10 años & 5,7 & 1,05 & 5,16 & 1,42 & 5,44 & 1,19 \\
\hline \multirow{2}{*}{ Jornada } & Parcial & 5,9 & 1,06 & 5,2 & 1,41 & 5,5 & 1,27 \\
& Completa & 6,9 & 1,17 & 5,5 & 1,22 & 6,1 & 1,09 \\
\hline
\end{tabular}

Nota. M=media, $D T=$ desviación típica, y un nivel de significación de $p<0,05$.

\section{Discusión}

De los resultados obtenidos se desprende, en primer lugar, que el perfil sociodemográfico del profesional de TA en Andalucía se caracteriza por estar casado o casada, con una edad media de 34 años, no tener una formación relacionada con el anexo VI del Decreto 22/2002 y una experiencia en la profesión de más de 10 años. El hecho que el 34,2\% de los encuestados manifestaron no tener titulación relacionado con el TA, supone un porcentaje similar al trabajo ofrecido por CamposIzquierdo (2004) del sector deportivo en Valencia, los cuales detecta un elevado número de trabajadores que desarrollan sus labores sin ninguna titulación relacionada con la actividad física y el deporte. Por ello, el presente estudio muestra la necesidad de regular el sector deportivo o TA, de lo contrario, el mercado laboral seguirá incorporando a profesionales poco cualificados, y en consecuencia podría perjudicar los servicios deportivos en el medio natural (Chamorro, 2010, Garrigos, 2002).

De este estudio destaca la elevada presencia de personas que trabajan por cuenta propia llegando casi a tres cuartas partes. Este dato verifica la tendencia creciente hacia la flexibilización del mercado laboral para dar respuesta a la necesidad de mano de obra en cada momento así como la necesidad de generar autoempleo (Aguirregabiria y Alonso-Borrego, 2009; Amadeo y Horton, 1997).

Sobre el género, encontramos estudios de proporciones similares como los de López-Viñaspre, Rodríguez y Boned (2003) en el sector de los gimnasios, los cuales ponen de manifiesto mayor presencia de hombres que mujeres, al igual otros del sector de la actividad física y el deporte (CEET, 2006; Telecyl, 2006). Sin embargo, encontramos proporciones opuestas sobre la paridad en los estudios de Boned et al., (2015) en trabajadores de centro fitness, o los de Alonso, Fernández, Gutiérrez y García (2004) sobre perfil del profesional de los centros deportivos de la ciudad de Vigo. Un estudio sobre la situación laboral de los titulados en Ciencias de la Actividad Física y el Deporte (Pérez et al., 2016) también se hace eco de una mayor proporción de hombres que de mujeres en todos 
los yacimientos de inserción que ofrece el mercado del deporte, incluyendo el de la actividad física en el medio natural. Este desequilibrio entre la proporción de hombres y mujeres en el mercado laboral parte de una menor presencia de alumnado femenino durante los estudios (Serra, 2016).

No se observa diferencias entre hombres y mujeres en las funciones de DT, MI y DTM, todo lo contrario que en estudios de otros ámbitos deportivos como los de Campos-Izquierdo, Martínez, Mestre, y Pablos (2007) sobre el perfil de los gestores deportivos, donde señalaban mayor presencia de hombres en cargos de responsabilidad, o los de Gallardo y campos (2011) sobre el perfil de trabajadores deportivos del ayuntamiento de Coslada (Madrid). El hecho de que tampoco se hayan encontrado diferencias entre el salario percibido deja entrever la inexistencia de una discriminación directa por la cual mujeres y hombres para un mismo recibirían remuneraciones distintas (Sallé y Molpeceres, 2010). En cambio, la dedicación desde el punto de vista del volumen de horas trabajado, sí que se presenta disimilitudes: los hombres, en mayor medida que las mujeres, trabajan a jornada completa. Una menor dedicación por parte de las mujeres puede explicarse por la sobrecarga del rol de la mujer. Las mujeres continúan dedicando más tiempo a la familia y al hogar que los hombres (Prieto \& Pérez de Guzmán, 2013). La Encuesta de Empleo del Tiempo realizada por el Instituto Nacional de Estadística para el período 2009-10, muestra como las mujeres dedican una media diaria de 4 horas y 4 minutos a la familia y el hogar, mientras que los hombres no llegan a la mitad del tiempo dedicado por ellas (INE, 2017).

En relación con la satisfacción laboral, las principales variables que inciden sobre esta son el ámbito laboral, dándose superior en los trabajadores del sector privado frente a los del sector público, y los años de experiencia. Desde la teoría del capital humano del economista Gary S. Becker (1993) se vincula los ingresos con la experiencia profesional y la edad de la persona. Es decir, con el paso del tiempo las personas adquieren una mayor experiencia que incrementa su capital humano y esto les lleva a conseguir mejores condiciones de trabajo e incrementar su salario.

No obstante, las variables salario percibido o tipo de contratación no se han presentado como condicionantes para el nivel de satisfacción. En el informe del proyecto VOCASPORT llevado a cabo por el Observatorio Europeo de Deporte y Empleo (Camy, Clijsen, Madella, y Pilkington, 2004) señalaba que las personas que trabajan en el ámbito deportivo sienten una gran devoción y por ello están dispuestas a sacrificarse en términos de retribución y condiciones laborales. Esta afirmación quedó constatada en el estudio sobre los titulados en Ciencias de la Actividad Física y el Deporte en Cataluña en el que se pudo comprobar que los egresados, a pesar de sentir que no recibían un salario justo respecto a su ocupación, presentaban un elevado nivel de satisfacción con su ocupación (Pérez, 2015).

De los resultados del presente estudio podemos concluir que los trabajadores de TA en Andalucía a modo general están satisfechos, ejercen su profesión por cuenta propia y son mayoritariamente hombres. Se presenta un sector que predomina el autoempleo dado que la mayor parte de los que desarrollan esta actividad lo hacen como autónomos. Con diferencias laborales entre hombres y mujeres, principalmente en el tipo de dedicación semanal, los hombres trabajan mayoritariamente a jornada completa, frente a las mujeres a jornada parcial. También el porcentaje de la jornada flexible es porcentualmente superior en los hombres. Aunque es común para todos los trabajadores que se desarrolle este servicio en horario "a demanda del cliente".

Del mismo modo, podemos decir que la satisfacción de estos trabajadores no guarda relación con las siguientes condiciones laborales: actividad desempeñada en la organización, tipo de contrato, realización de otras funciones propias de la actividad, horas de dedicación semanal o sueldo percibido.

Por último, se ha observado que la mayoría de los trabajadores no tienen una formación relacionada con el anexo VI del Decreto 22/2002 de 26 de enero, de turismo en el medio rural y TA. Por este motivo, los resultados obtenidos en el presente estudio pueden servir de base para llevar a cabo una adecuada regulación profesional en el sector del TA en Andalucía por los organismos competentes. De la misma manera, se recomiendan futuras investigaciones tanto para ampliar el conocimiento sobre la satisfacción laboral de los trabajadores de TA como sus condicionantes. 


\section{Referencias}

Aamodt, M. G. (2010). Psicología Industrial/Organizacional. Un enfoque aplicado. México: Thomson Paraninfo.

Aguirregabiria, V. y Alonso-Borrego, C. (2009). Labor contracts and flexibility: evidence from a labor market reform in Spain. Working Papers, 11, 1-32.

Alonso, D., Fernández, R., Gutiérrez, A., y García, J. L. (2004). Perfil del Profesional de los Centros Deportivos de la Ciudad de Vigo (Galicia). Recuperado de http://www.cienciadeporte.com/congreso/04\%20val/pdf/c67.pdf

Alonso, D., Fernández, R., Gutiérrez, A., Y García, J.L. (marzo, 2004). Perfil del profesional de los centros deportivos de la ciudad de Vigo. Comunicación presentada en el III Congreso de la Asociación Española de Ciencias del Deporte, Valencia.

Amadeo, E. y Horton, S. (1997). Labour productivity and flexibility. New York: Macmillan Press Ltd.

Anaya, D., y Suárez, J. M. (2004). La escala de satisfacción laboral - Versión para orientadores (ESL-VO) como recurso para la evaluación de la satisfacción laboral. Revista de Investigación Educativa, 22(2), 219224.

Barranco, D., Grimaldi-Puyana, M., Crovetto, M., Barbado, C., Boned, C., y Felipe, J.L. (2015). Diferencias laborales entre gestores deportivos con y sin titulación universitaria en ciencias de la actividad física y el deporte. Journal of sport and health research, 7(2), 81-90.

Becker, G. S. (1993). Human Capital (3a ed.). Chicago: The University of Chicago Press, Chicago.

Bernabé, B., Campos-Izquierdo, A., y González, M. (2016). Job satisfaction of sport and physical activity instructors in Spain according to gender and age. South African Journal for Research in Sport, Physical Education and Recreation, 38(1) 1-12.

Campos, A. (2004). Situación profesional de las personas que trabajan en funciones de actividad física y deporte en la Comunidad Autónoma Valenciana. (Tesis Doctoral). Facultat de Ciències de l'Activitat Física i 1’Esport, Universitat de València, Valencia.

Campos, A., Martínez, J., Mestre, J.A., y Pablos, C. (2007). Los profesionales de la organización y gestión de actividad física y deporte en las instalaciones deportivas y entidades: características socio-demográfica. Revista Internacional de Ciencias del Deporte. 8(3), 25-38.

Camy, J., Clijsen, L., Madella, A. y Pilkington, A. (2004). Vocasport. Improving employment in the field of sport in Europe through vocational training. Vocational education and training in the field of sport in the European Union: situation, trends and outlook. Lyon: European Network of Sport Science, Education and Employment, Université Claude Bernard.

Casas, J., Repullo, J. R.; Lorenzo, S., Cañas, J. J. (2002). Dimensiones y medición de la calidad de vida laboral en profesionales sanitarios. Revista de Administración Sanitaria, 6(23), 143-160.

Centro de Estudios Económico Tomillo. (2006). La familia profesional de las Actividades Físicas y Deportivas. Estudio desarrollado para el INCUAL. Documento sin publicar.

CSD (2015). Encuesta de hábitos deportivos en España 2015. Madrid: Ministerio de Educación Cultura y Deporte. Revista Internacional de Ciencias del Deporte, 8(3), 25-38.

Gallardo, J. y Campos, A. (2011). Situación profesional de los recursos humanos de la actividad física y el deporte en el municipio de Coslada. Revista Internacional de Medicina y Ciencias de la Actividad Física y el Deporte, 11(43), 440-454.

Gambau, V. (2011). Deporte y empleo en España: dificultades de estudio y de intervención. Revista Española de Educación Física y el Deporte, 394, 13-36.

García-Saura, P.J. (200). Turismo activo y medio ambiente: una implicación a aspectos jurídicos. Asociación Científico Cultural en Actividad Física y Deporte, 1, 79-98.

Garrigos, F. (2002). La regulación profesional como activo en el desarrollo de la economía y el deporte. Actividad Física y Deporte, (2) 5-8.

González-Molina, A. (2008). Rasgos caracterizadores del turismo activo y turismo deportivo e importancia económico-social y estructural de nuevas formas emergentes. Asociación Científico Cultural en Actividad Física y Deporte, 1, 79-98

Grimaldi-Puyana, M. (2015). Análisis de las condiciones laborales de los gestores deportivos en puestos intermedios. Actividad Física y Deporte: ciencia y profesión, 23(2), 13-18. 
Grimaldi-Puyana, M., Felipe, J., y Cuadrado-Reyes, J. (2013). Adaptación en la organización y gestión de los riesgos en las actividades físico deportivas en el medio natural. Revista habilidad motriz, 40, 26-33.

Grimaldi-Puyana, M., Fernández-Gavira, J., y García-Fernández, J. (2013). Situación laboral de los gestores deportivos en servicios públicos externalizados. Actividad Física y Deportes: Ciencia y Profesión, 18, $33-40$.

Gutiérrez-Pérez, J. (2003). Competencias del Monitor de Naturaleza en los Espacios Naturales Protegidos. Sevilla: Junta de Andalucía.

INE (2017). Encuesta de Empleo del Tiempo (EET). Recuperado de http://www.ine.es/prensa/eet_prensa.htm

López-Viñaspre, P. Rodríguez, G., y Boned, C. (2003). Los recursos humanos en el sector del fitness. Una aproximación al panorama actual. Instalaciones Deportivas XXI, 126, 38-46.

Luque-Gil, M. (2008). La evaluación del medio para la práctica de actividades turístico-deportivas en la naturaleza. Cuadernos de turismo, 12, 131-149

Martín, M., Campos-Izquierdo, A., Jiménez, J. E., y Martínez, J. (2007). Calidad de vida y estrés laboral: la incidencia del burnout en el deporte de alto rendimiento madrileño. Revista Internacional de Ciencias del Deporte, 6, 64-77.

Pérez, M. (2015). El mercat de treball de les persones llicenciades en Ciències de l'Activitat Física i l'Esport a Catalunya. (Tesis doctoral). Universidad de Barcelona, Facultad de Educación, Barcelona.

Pérez, M., Vilanova, A., y Grimaldi-Puyana, M. (2016). Mercado actual de trabajo de los graduados en Ciencias de la Actividad Física y el Deporte: Mirada hacia el autoempleo. Journal of Sports Economics \& Management, 6(3), 149-162.

Pérez, M., y Vilanova, A. (2015). El mercat de treball de les llicenciades en Ciències de l'Activitat Física i l'Esport. En Quadern VIII Dones i Esport. Institut Barcelona Esports, Barcelona.

Prieto, C., y Pérez de Guzmán, S. (2013). Desigualdades laborales de género, disponibilidad temporal y normatividad social. Revista Española de Investigaciones Sociológicas, 141, 113-132.

Rebollo-Rico, S. y Latiesa, M. (2002). Salidas Profesionales en el campo del turismo activo. Sevilla: Junta de Andalucía.

Robbins, S. P. (2004). Comportamiento organizacional. México: Pearson Educación.

Salguero-Pérez (2002). Los recursos naturales como atractivo. En Salidas Profesionales en el campo del turismo activo (pp101-113). Sevilla: Junta de Andalucía.

Sallé, M., y Molpeceres, L. (2010). La Brecha salarial: realidades y desafíos. Las desigualdades salariales entre mujeres y hombres España 2009. Madrid: Ministerio de Igualdad y Ministerio de Trabajo e Inmigración.

Sánchez S. M. (2006). Diseño de un modelo causal de satisfacción laboral: Aplicación en el sector servicios. Tesis Doctoral. Córdoba: Universidad de Córdoba.

Sánchez-Alcaraz, B. J., Gómez, A., Parra, M. ${ }^{a}$ C., y García, J. A. (2014). Análisis de la satisfacción laboral de técnicos deportivos de pádel y natación. Apunts Educación Física y Deportes, 117(3), 77-83.

Sánchez-Alcaraz, B.J. (2012). Nivel de satisfacción laboral de los socorristas de playa. EmasF, 4(19), 130-137.

Sánchez-Alcaraz, B.J., y Parra-Meroño, M.C. (2013). Diseño y validación de un cuestionario de satisfacción laboral para técnicos deportivos (CSLTD). Revista cultura ciencia y Deporte, 9(8) 199-129.

Sánchez-Oliver, A. J., García-Fernández, J., Grimaldi-Puyana, M., y Feria, A. (2017). Oportunidades de emprendimiento: Evaluación de las tendencias relacionadas con la actividad física y el deporte del ACSM. Journal of Sports Economics \& Management, 7(1), 2-13.

Serra, P. (2016). La perspectiva de género en los estudios de ciencias de la actividad física y el deporte. (Tesis doctoral). Universidad de Barcelona, Facultad de Educación, Barcelona.

Sierra, R. (2001). Técnicas de Investigación Social: Teoría y Ejercicios. Madrid: Editorial Paraninfo.

Telecyl Estudios. (2006). Estudio sectorial: Actividades deportivas y sus instalaciones-principales conclusiones. Estudio realizado por la Fundación Tripartita para la formación en el empleo. Documento sin publicar.

Theriou, N., y Chatzoglou, P. (2008). Enhancing performance through best HRM practices, organizational learning and knowledge management. European Business Review, 20(3), 185-207.

Warr, P., Cook, J., y Wall, T. (1979). Scales for the measurement of some work attitudes and aspects of psychological well-being. Journal of Occupational Psychology, 52, 129-14. 